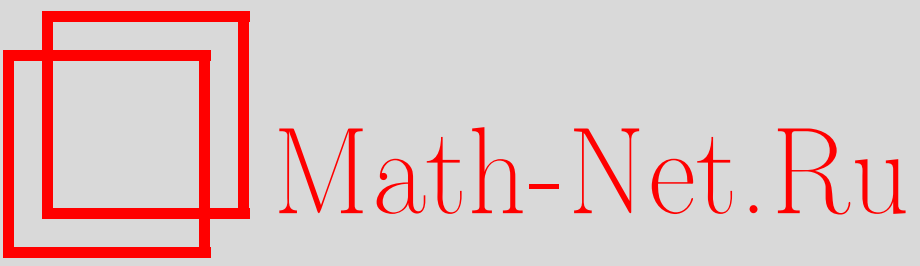

Л. Д. Пустыльников, Обобщенный ньютоновский периодический биллиард в шаре, УМH, 2005, том 60, выпуск 2, 171-172

DOI: https://doi.org/10.4213/rm1420

Использование Общероссийского математического портала Math-Net.Ru подразумевает, что вы прочитали и согласны с пользовательским соглашением

http://www.mathnet.ru/rus/agreement

Параметры загрузки:

IP: 52.6 .47 .48

26 апреля 2023 г., 04:44:10 


\title{
ОБОБЩЕННЫЙ НЬЮТОНОВСКИЙ ПЕРИОДИЧЕСКИЙ БИЛЛИАРД В ШАРЕ
}

\author{
Л. Д. Пустыльников
}

Обобщенные биллиарды в произвольной области $B$ были введены в [1], а в случае, когда область $B$, внутри которой массивная точка двигается прямолинейно с постоянной скоростью, есть параллелепипед, такие биллиарды были введены в [2] и изучались в рамках ньютоновской механики в [1] и [2] (ньютоновский биллиард) и в рамках релятивистской механики в [1]-[4] (релятивистский биллиард). Существо обобщения состоит в том, что при отражении точки от границы $\Gamma$ области $B$ проекция $v_{\tau}$ ее скорости $v$ на касательное пространство к $\Gamma$ в точке отражения не меняется, а проекция $v_{\nu}$ ее скорости $v$ на нормалњ в точке отражения преобразуется с помощью заранее заданной функции $f(\gamma, t)$, определенной на прямом произведении $\Gamma \times \mathbb{R}^{1}\left(\mathbb{R}^{1}\right.$ - прямая линия, $\gamma \in \Gamma$ - точка границы $\Gamma$, а величина $t \in \mathbb{R}^{1}$ обозначает время) согласно следующему закону. Предположим, что траектория точки, которая движется со скоростью $v$, пересекает $\Gamma$ в точке $\gamma \in \Gamma$ в момент времени $t^{*}$. Тогда в этот момент времени $t^{*}$ точка приобретает такую скорость $v^{*}$, как если бы она подверглась упругому удару с бесконечно-тяжелой плоскостью $\Gamma^{*}$, касающейся $\Gamma$ в точке $\gamma$, которая двигается в момент времени $t^{*}$ вдоль нормали к $\Gamma$ в точке $\gamma$ со скоростью $(\partial f / \partial t)\left(\gamma, t^{*}\right)$. При этом упругий удар рассматривается в рамках ньютоновской механики, а в качестве положительного направления движения плоскости $\Gamma^{*}$ выбирается направление внутрь области $B$. В настоящей работе изучается частньй случай обобщенного ньютоновского биллиарда, в котором область $B$ есть $n$-мерньй $(n \geqslant 1)$ шар радиуса $R$, граница $\Gamma$ $(n-1)$-мерная сфера, а $f(\gamma, t)=f(t)$ - бесконечно-дифференцируемая функция, не зависящая от $\gamma$ и имеющая период $2 \pi$ по $t$, т.е. $f(t)=f(t+2 \pi)$.

Теорема. Предположим, что в начальный момент времени $t_{0}$ точка имела скорость $v_{0}$. Тогда во все последующие моменты времени $t \geqslant t_{0}$ модуль скорости $|v|$ точки не превосходит константу, зависящую только от $v_{0}, R$ и функции $f(t)$.

ДокАЗАТЕЛЬСтво. Предположим, что в некоторьй момент времени $t$ столкновения точки с границей $\Gamma$ в положении $\gamma \in \Gamma$ точка приобрела скорость $v=\left(v_{\tau}, v_{\nu}\right)$, направленную внутрь $B$, а в ближайший к $t$ момент времени $t^{\prime} \geqslant t$ столкновения с Г в положении $\gamma^{\prime} \in \Gamma$ точка приобрела скорость $v^{\prime}=\left(v_{\tau}^{\prime}, v_{\nu}^{\prime}\right)$. Здесь $v_{\tau}$ и $v_{\tau}^{\prime}-$ соответственно проекции векторов $v$ и $v^{\prime}$ на касательные пространства к Г в точках $\gamma$ и $\gamma^{\prime}$, а $v_{\nu}$ и $v_{\nu}^{\prime}$ - соответственно координаты проекций векторов $v$ и $v^{\prime}$ на нормали к Г в точках $\gamma$ и $\gamma^{\prime}$. Из определения обобщенного ньютоновского биллиарда следует, что

$$
\left|v_{\tau}\right|=\left|v_{\tau}^{\prime}\right|, \quad t^{\prime}=t+\frac{2 R v_{\nu}}{v_{\nu}^{2}+\left|v_{\tau}\right|^{2}}, \quad v_{\nu}^{\prime}=v_{\nu}+2(d f / d t)\left(t^{\prime}\right)
$$

Рассмотрим отображение $A:\left(t, v_{\nu}\right) \rightarrow\left(t^{\prime}, v_{\nu}^{\prime}\right)$ и введем новые переменные $r$ и $r^{\prime}$ так, что

$$
r=\frac{2 R v_{\nu}}{\varepsilon\left(v_{\nu}^{2}+\left|v_{\tau}\right|^{2}\right)}, \quad r^{\prime}=\frac{2 R v_{\nu}^{\prime}}{\varepsilon\left(\left(v_{\nu}^{\prime}\right)^{2}+\left|v_{\tau}^{\prime}\right|^{2}\right)},
$$

где $\varepsilon>0$ - малый параметр. Рассматривая переменные $t$ и $r$ как полярные координаты $(t-$ угол, $0 \leqslant t<2 \pi, r$ - радиус) и выражая через них отображение $A$, получим отображение $U_{\varepsilon}:(t, r) \rightarrow$ $\left(t^{\prime}, r^{\prime}\right)$, имеющее в силу (1) и (2) следующий вид:

$$
t^{\prime}=t+\varepsilon r \bmod 2 \pi, \quad r^{\prime}=r+\varepsilon r \varphi(t, r, \varepsilon),
$$

где

$$
|\varphi(t, r, \varepsilon)|<c r
$$

Работа выполнена при частичной поддержке РФФИ (грант 02-01-01067). 
$c$ - константа, не зависящая от $t, r$ и $\varepsilon$. Так как преобразование $A$ сохраняет площадь, то для отображения $U_{\varepsilon}$ справедливо следующее свойство: любая замкнутая несамопересекающаяся кривая, окружающая точку $r=0$, пересекается со своим образом относительно действия $U_{\varepsilon}$. Поэтому в силу соотношений (3) и (4) существуют такие константы $\varepsilon_{0}>0$ и $r_{0}>0$, что при всех $\varepsilon \leqslant \varepsilon_{0}$ в области $0<r \leqslant r_{0}$ справедливо утверждение теоремы Мозера [5], согласно которому в этой области существует замкнутая несамопересекающаяся кривая П, окружающая точку $r=0$ и инвариантная относительно действия $U_{\varepsilon}$. Так как в этом случае область внутри кривой П также инвариантна относительн $U_{\varepsilon}$, то, переходя обратно к координате $v_{\nu}=(r \varepsilon)^{-1}$, получим, что при любом сколь угодно болшшом значении величины $\left|v_{\nu}\right|$ траектория точки $\left(t, v_{\nu}\right)$ относительно отображения $A$ не вьйдет за пределы некоторого круга с центром в точке $v_{\nu}=0$. Теорема доказана.

\section{СПИСОК ЛИТЕРАТУРЫ}

[1] Л. Д. Пустыльников // УМН. 1999. Т. 54. №3. С. 179-180. [2] Л. Д. Пустыльников // УМН. 1995. Т. 50. № 1. C. 143-186. [3] M. V. Deryabin, L. D. Pustyl'nikov // Lett. Math. Phys. 2003. V. 63. № 3. P. 195-207. [4] M. V. Deryabin, L. D. Pustyl'nikov // Regul. Chaotic Dyn. 2003. V. 8. №3. P. 283-296. [5] J. K. Moser // Nachr. Akad. Wiss. Göttingen Math.-Phys. Kl. II. 1962. P. 1-20.

Институт прикладной математики

Представлено Р. А. Минлосом им. М. В. Келдыша РАН 